\title{
Fracturas de los huesos del carpo
}

\author{
F. García de Lucas, J. M. Abad, P. J. Delgado, \\ A. Fuentes, J. I. García López
}

Unidad de Miembro SuPERIor Hospital FREMAP. Majadahonda (Madrid)

Correspondencia:

fernando_garcia@fremap.es

\section{INTRODUCCIÓN}

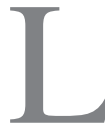

as fracturas de los huesos del carpo son lesiones frecuentes que se originan en la mayoría de los casos tras una caída sobre la mano con la muñeca en extensión. Las más comunes son las que afectan al escafoides, seguidas de las fracturas del piramidal, siendo las menos frecuentes las del trapezoide ${ }^{1}$.

\section{FRACTURAS DEL ESCAFOIDES}

Las fracturas del escafoides son las más frecuentes de las fracturas del carpo $(80 \%) \mathrm{y}$, después de las del radio distal, las segundas en frecuencia de la extremidad superior. Los síntomas pueden ser mínimos y pueden no ser diagnosticadas en la fase aguda; con frecuencia se diagnostican como esguince de muñeca, pero la persistencia de dolor y tumefacción en la tabaquera anatómica y/o en la zona dorsal y radial de la muñeca debe alertarnos sobre su existencia. El $70 \%-80 \%$ de las fracturas se localizan en el tercio medio, el $10 \%-20 \%$ en el polo proximal y el $10 \%$ en el polo distal ${ }^{1}$. En el estudio epidemiológico realizado por Hove en Noruega, se observó que el $82 \%$ de las fracturas ocurren en hombres, con una mayor incidencia entre los 20 y los 30 años ${ }^{2}$.

El escafoides posee una morfología irregular y es el eslabón fundamental en la biomecánica del carpo. El centro de la superficie articular distal es palmar con respecto a la superficie articular proximal de forma que se produce un momento de flexión cuando las fuerzas axiales atraviesan la muñeca en dirección al antebrazo.
Las fuerzas de flexión sobre el polo distal y las de extensión sobre el polo proximal son la causa de la deformidad en joroba (Figura 1) que aparece en las seudoartrosis de escafoides ${ }^{3}$.

La vascularización del escafoides se realiza a través de dos sistemas, el primero y más importante por la cresta dorsal y radial del escafoides y el segundo volar y limitado a la tuberosidad ${ }^{4}$.

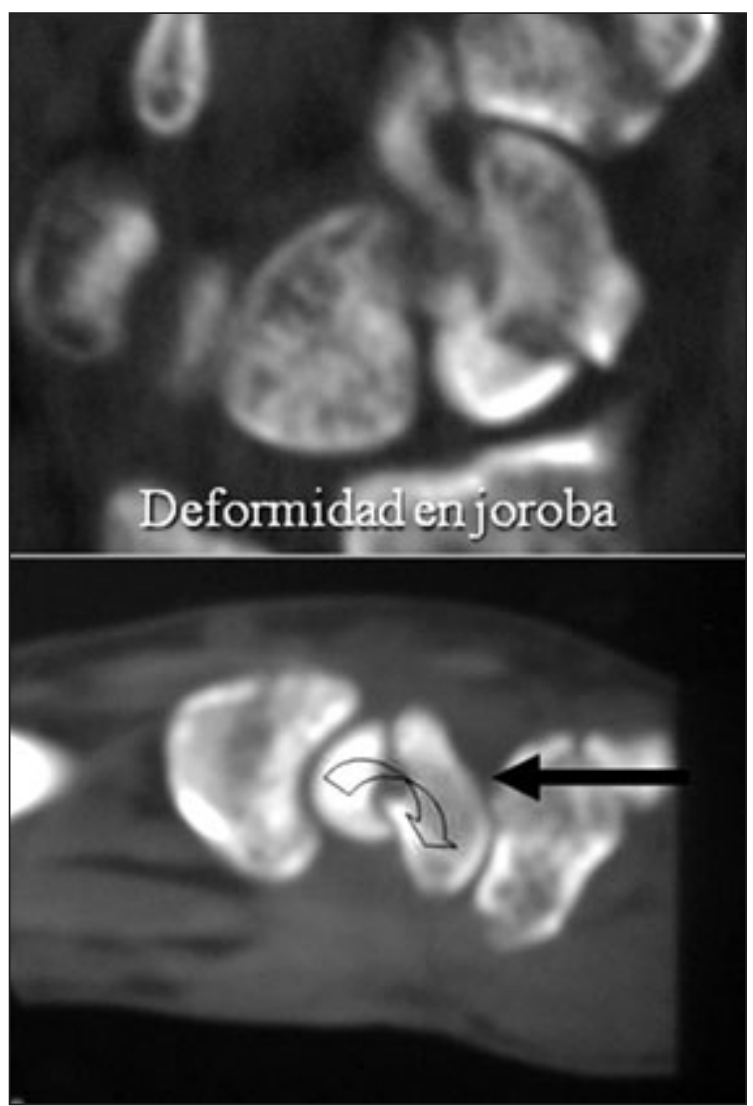

Figura 1: Deformidad en joroba del escafoides. El polo proximal del escafoides se coloca en extensión. El polo distal se flexiona, se desvía hacia ulnar y se coloca en pronación. 
La nutrición intraósea desde distal a próximal deja al polo proximal en una situación precaria que predispone a la necrosis avascular a medida que el trazo de fractura se localiza en zonas proximales, pues ni el ligamento radio-escafoides-semilunar de Testuz-Kuentz ni el ligamento escafoides-semilunar proporcionan suficiente aporte vascular.

\section{¿Cómo se producen?}

Como consecuencia de una caída con la muñeca en hiperextensión superior a $\operatorname{los} 90^{\circ}$ en la que se produce una fuerza de reacción que impacta el escafoides con el radio distal ${ }^{5}$. Debido a que el escafoides es un hueso que está cubierto por cartílago hialino prácticamente en toda su superficie, las fracturas no siempre se acompañan de clínica llamativa (dolor en tabaquera anatómica y/o en el borde dorso-radial de la muñeca, edema e hipersensibilidad a nivel de la tabaquera anatómica y dolor al realizar la compresión axial en el eje del primer radio).

\section{¿Cómo se diagnostican?}

Ante la sospecha de una fractura del escafoides se debe realizar un estudio radiológico, cuya proyección más adecuada es una posteroanterior de la muñeca con inclinación cubital y una supinación de $45^{\circ}$ (Figura 2). Con esta posición de la muñeca, el escafoides se expone con su mayor longitud de forma perpendicular al haz de rayos $\mathrm{X}$ y disminuye la incidencia de falsos negativos. Si existe duda de la existencia de una fractura y se dispone de una TAC o una RM, ambas nos proporcionarán un diagnóstico fiable y precoz. Si no es así, es recomendable repetir el estudio radiológico al cabo de dos semanas, donde la necrosis de los bordes de la fractura permitirá realizar el diagnóstico con más facilidad.

El análisis debe permitirnos conocer la estabilidad o inestabilidad de la fractura y orientarnos hacia el tratamiento adecuado a cada paciente. Probablemente la clasificación de las fracturas de escafoides más utilizada en nuestros días sea la descrita por Herbert y Fischer en $1984^{6}$ (Figura 3) sin que exista una buena reproducibilidad inter e intraobservador ${ }^{7}$. Para estos autores, las fracturas de la tuberosidad y las del tercio medio incompletas son estables (A1 y A2), el resto son

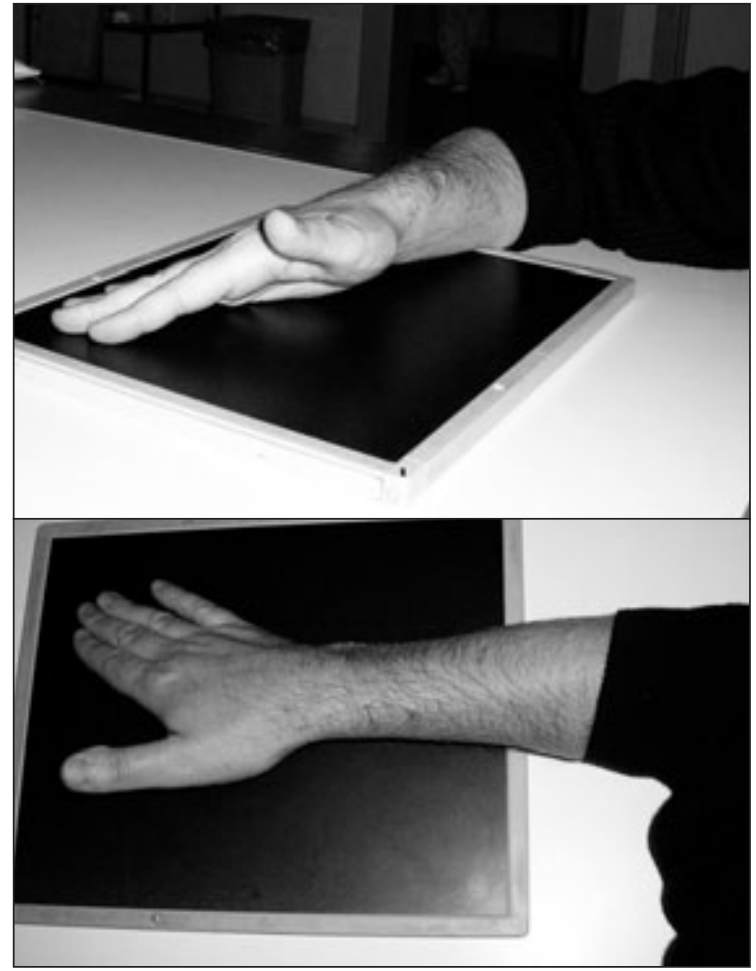

Figura 2: Posición de la muñeca más adecuada para visualizar una fractura de escafoides mediante estudio radiológico.

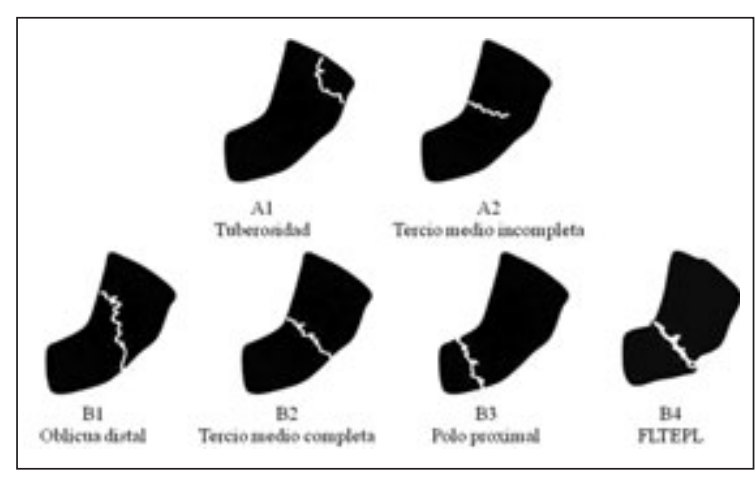

Figura 3: Clasificación alfanumérica realizada por Herbert y Fischer que combina trazo de fractura, estabilidad y cronicidad ${ }^{6}$. (FLTEPL: fractura-luxación transescafoperilunar).

inestables (B1, B2, B3 y B4). Desde un punto de vista práctico y atendiendo a parámetros aceptados globalmente, se pueden considerar fracturas estables aquellas en las que no existe o es mínimo el desplazamiento entre los fragmentos, y fracturas inestables las que presentan un desplazamiento mayor de $1 \mathrm{~mm}$, angulación anormal entre los fragmentos y/o alineación inadecuada del carpo. 


\section{¿Cómo se tratan?}

Las fracturas estables se pueden tratar mediante inmovilización con yeso antebraquial durante 8-12 semanas consiguiendo la consolidación en un $85-90 \%{ }^{8}$. La posición de la muñeca no influye en el resultado final, como tampoco lo hace la inclusión o no del pulgar. La valoración de la consolidación del escafoides es motivo de controversia, aumentado la fiabilidad la utilización de la TAC o la RM, cuya interpretación en ocasiones hace dudar acerca de si los puentes óseos encontrados son o no suficientes para considerar la fractura consolidada ("la TAC desnuda la fractura"). Aceptando que el escafoides es la pieza fundamental en la biomecánica del carpo, el objetivo del tratamiento de las fracturas será doble, la consolidación y que ésta sea anatómica, sin deformidad residual. Es por ello que la osteosíntesis percutánea de las fracturas estables se debe consi-
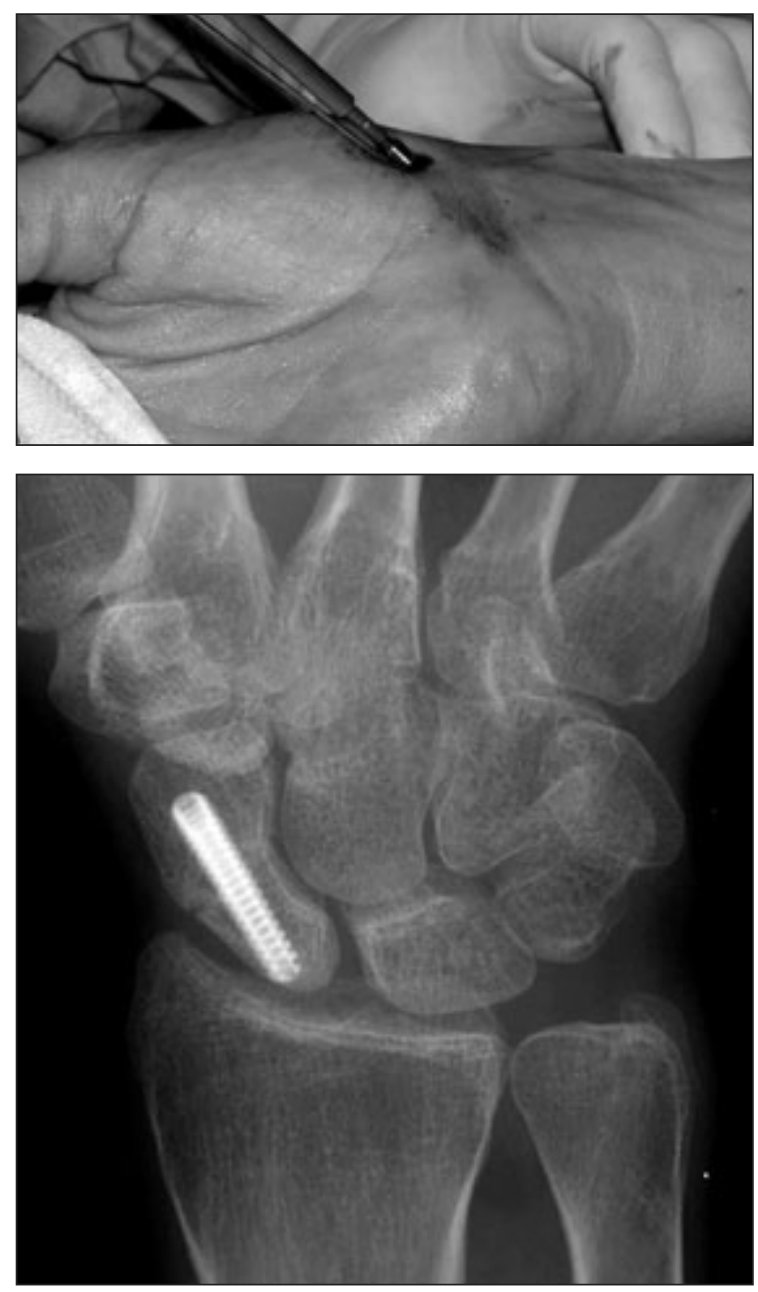

derar como alternativa terapéutica con índices de consolidación iguales o superiores al tratamiento conservador. El paciente debe ser informado de que a pesar de los riesgos que supone (gesto quirúrgico con complicaciones potenciales), tiene ventajas considerables (asegura la estabilidad, evita el desplazamiento de los fragmentos, disminuye el tiempo de inmovilización y supone una mejor relación coste-beneficio) $)^{9-12}$. En las fracturas del tercio medio, la osteosíntesis percutánea puede realizarse a través de un abordaje dorsal o volar (Figura 4); en las del polo proximal o las localizadas en el tercio distal es preferible utilizar un abordaje dorsal (Figura 5). Este último permite colocar el tornillo perpendicular al trazo de fractura mientras que el abordaje volar colocará el tornillo en una dirección oblicua con respecto a la fractura, sin que ello suponga inconvenientes desde un punto de vista clínico.
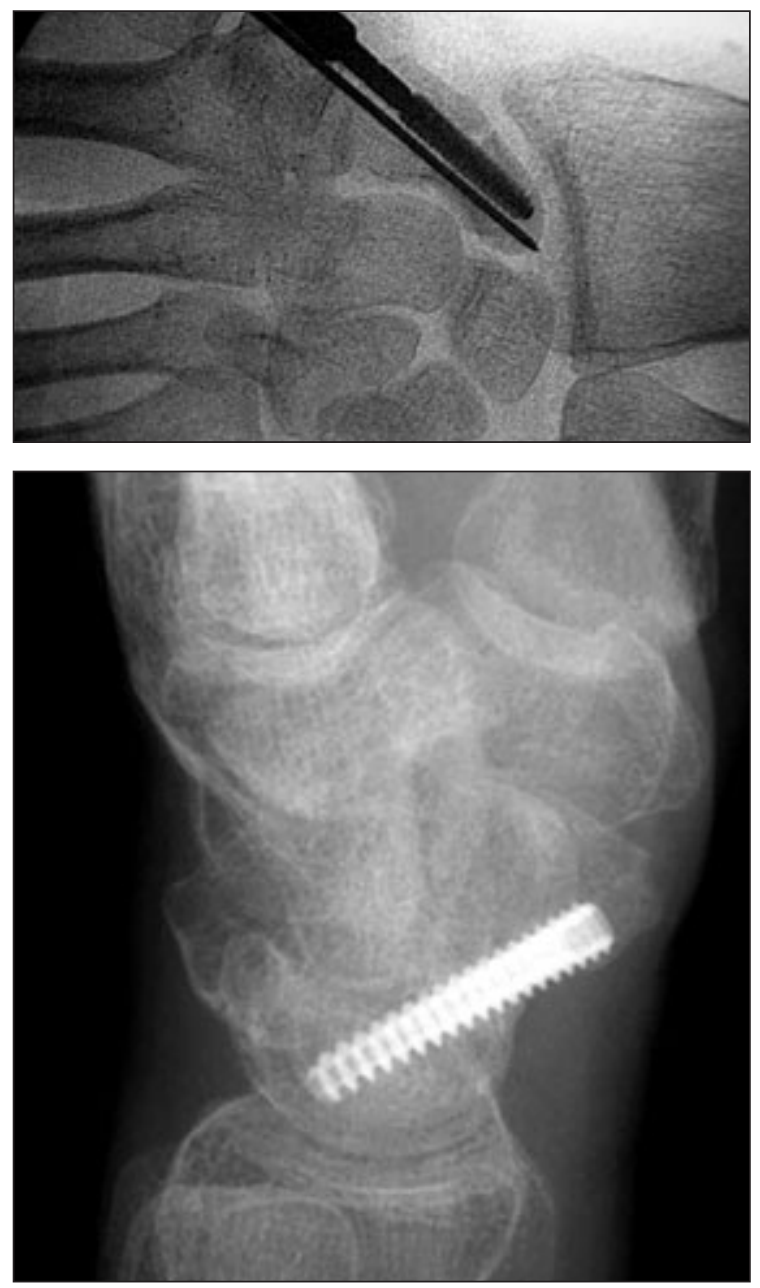

Figura 4: Osteosíntesis percutánea por vía volar. 

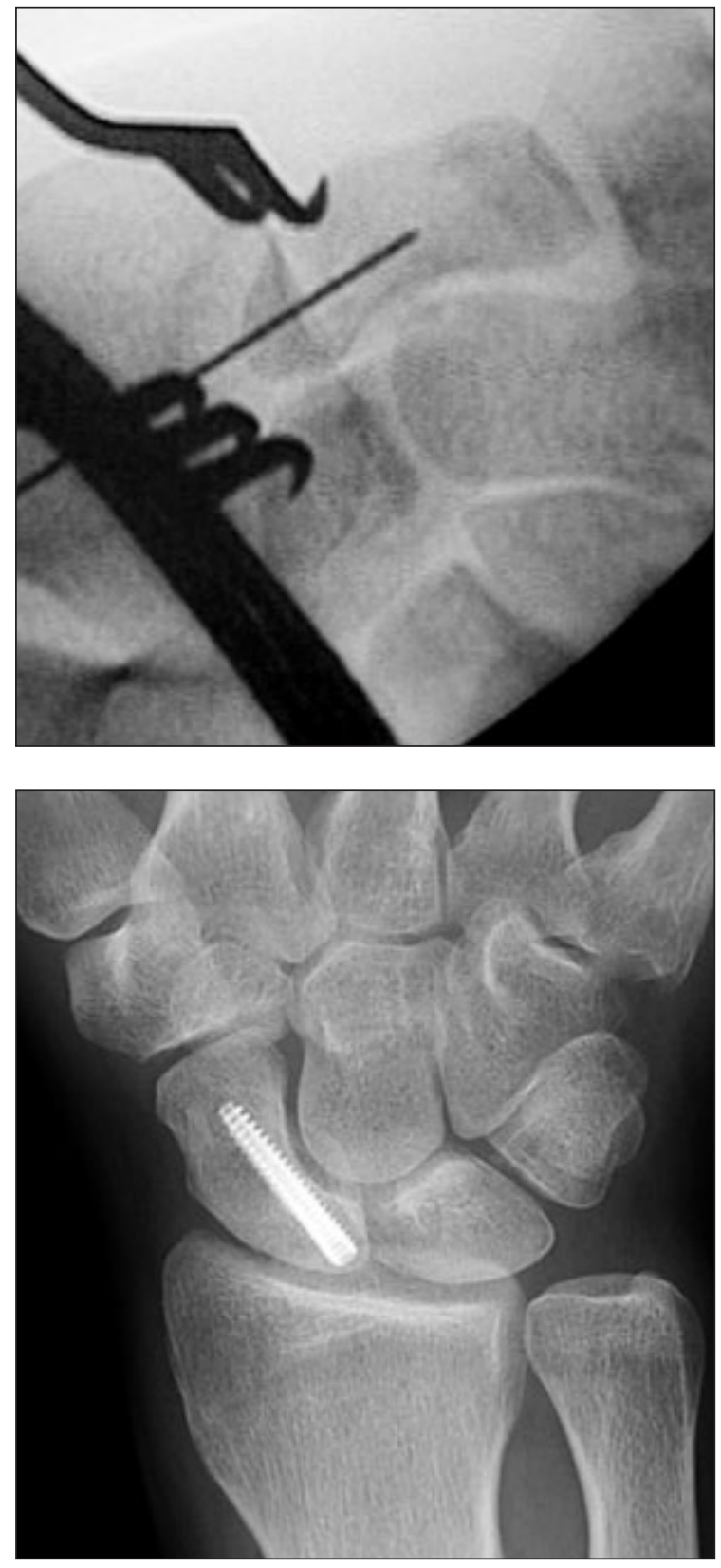
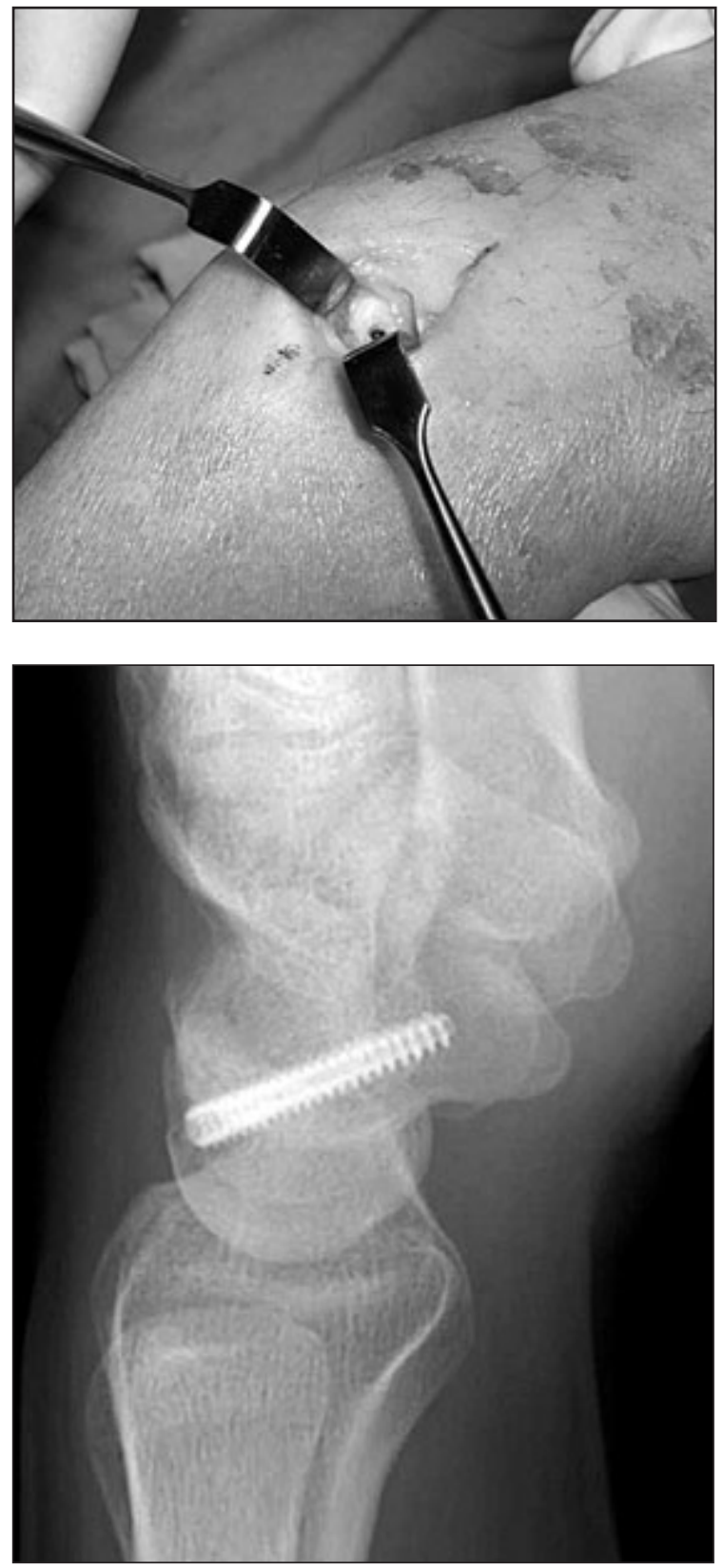

Figura 4: Osteosíntesis percutánea por vía dorsal.

Las fracturas inestables son objeto de tratamiento quirúrgico mediante reducción cerrada o abierta y osteosíntesis con agujas de Kirschner o tornillos, siendo preferible esta última fijación si el trazo de fractura lo permite ${ }^{13}$. La reducción abierta, indicada en las fracturas en las que no es posible conseguir una reducción adecuada y estable con la manipulación cerra$\mathrm{da}$, tiene riesgos potenciales (mayor incidencia de rigidez de muñeca, lesión de ligamentos radio-carpianos extrínsecos y lesión de tendones extensores) que se soslayan con la osteosíntesis percutánea, no siempre posible en fracturas inestables. Las indicaciones para realizar un abordaje dorsal o volar son superponibles a las mencionadas en la osteosíntesis percutánea con las siguientes matizaciones: en el caso de las fracturas-luxaciones, el abordaje dorsal permite reparar las lesiones ligamentosas asociadas y, en caso de tener que aportar injerto óseo para conseguir una mayor estabilidad, es preferible el abordaje volar. 


\section{FRACTURAS DEL SEMILUNAR}

Las fracturas del semilunar, no relacionadas con la enfermedad de Kienböck, son infrecuentes. Teisen y Hjarbaek ${ }^{14}$ diagnosticaron 17 fracturas y 93 necrosis del semilunar durante un periodo de 31 años en la misma institución hospitalaria. Ninguna de las 17 fracturas evolucionaron a necrosis, y las clasificaron en cinco tipos. Tipo I (polo volar), las más frecuentes, nueve casos; debido a que los ligamentos estabilizadores más importantes del semilunar se encuentran en la cara volar y a que la mayoría de los vasos nutricios penetran por esta cara, es recomendable estabilizar estas fracturas con Kirschner o tornillos canulados para minimizar el riesgo de necrosis y de inestabilidad.
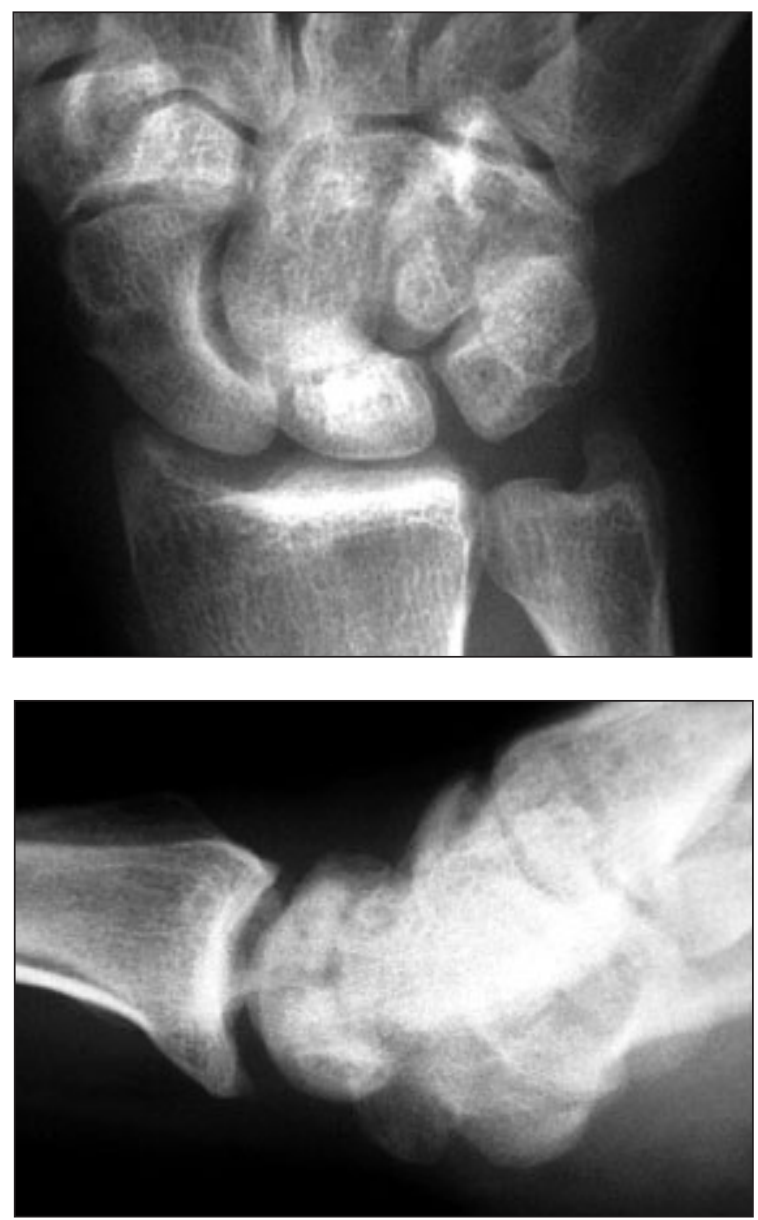

Tipo II (marginales-arrancamiento), cuatro casos; Tipo III (polo dorsal), dos casos y Tipo IV (fractura en el plano sagital), un caso; en estos tres tipos de fractura el tratamiento recomendado es la inmovilización durante 4-6 semanas. Tipo $V$ (fractura en el plano coronal), un caso; se recomienda la fijación con Kirschner o tornillos canulados (Figura 6).
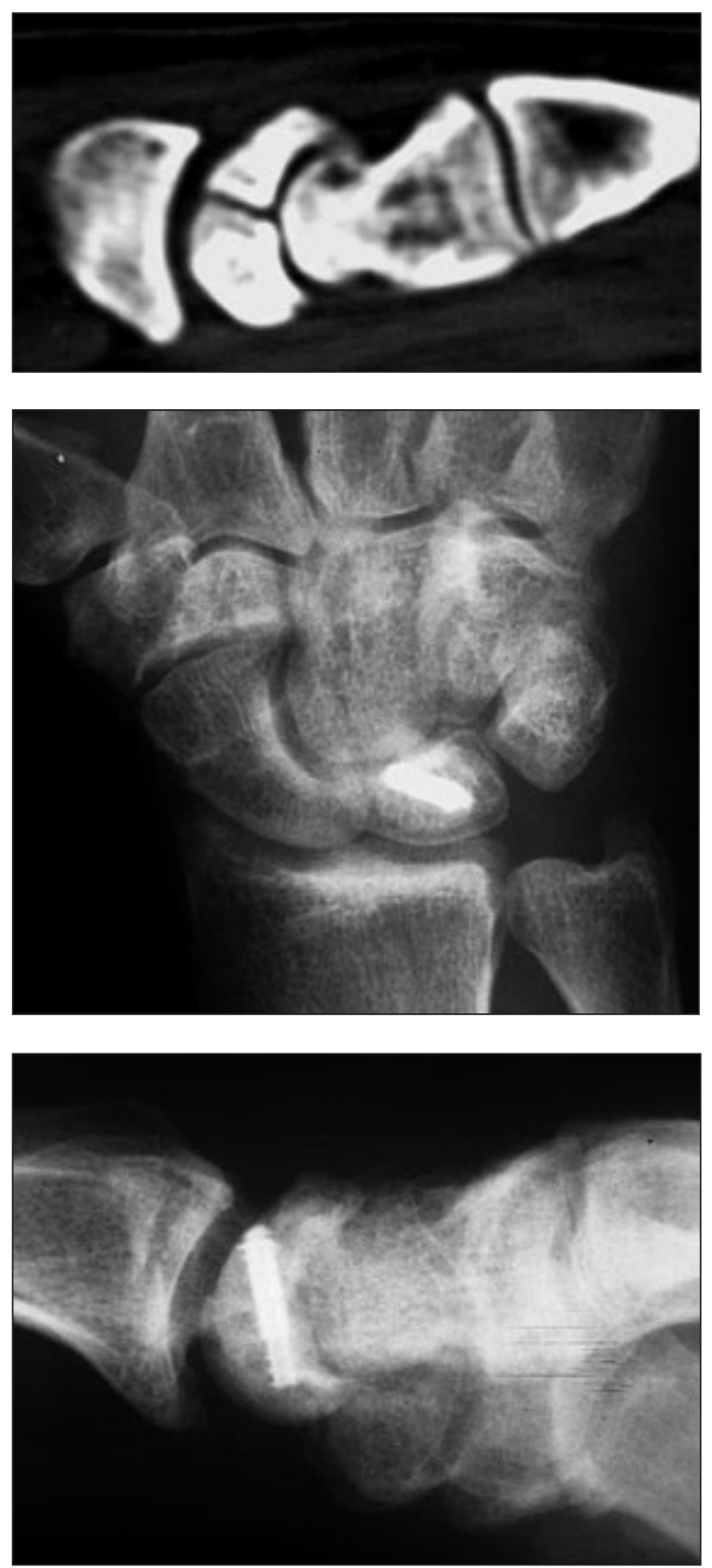

Figura 6: Fractura del semilunar en el plano coronal (Tipo V de la clasificación de Teisen y Hjarbaek ${ }^{14}$ ) tratada con tornillo canulado. 


\section{FRACTURAS DEL PIRAMIDAL}

Constituyen el segundo grupo más frecuente y suelen estar asociadas a otras lesiones del carpo. Se clasifican en tres tipos ${ }^{15,16}$. Fracturas corticales dorsales como consecuencia de un mecanismo de avulsión o cizallamiento o impactación contra la estiloides cubital; a pesar de que el fragmento cortical puede no unir, frecuentemente son asintomáticas y sólo de forma excepcional requieren la escisión del fragmento. En los casos de avulsión por los ligamentos dorsales escafoides-piramidal y radio-piramidal, la fractura puede representar una forma frustrada de luxación perilunar. Causan poca morbilidad y con 6 semanas de inmovilización se consigue un buen resultado. Fracturas del cuerpo, menos frecuentes que la fracturas corticales dorsales, si son lesiones aisladas, habitualmente no están desplazadas y requieren inmovilización durante 4-6 semanas. Analizar correctamente los estudios de diagnóstico por imagen, pues pueden formar parte de una luxación perilunar (estadio III de la inestabilidad perilunar de Mayfield ${ }^{17}$ ). Fracturas volares por avulsión, no son frecuentes pero pueden representar un signo de inestabilidad por avulsión del ligamento cúbito-piramidal; el tratamiento es objeto de controversia y probablemente la premisa es tratar la inestabilidad y no la fractura en sí.

\section{FRACTURAS DEL PISIFORME}

El mecanismo más frecuente es un impacto directo sobre la eminencia hipotenar pero los traumatismos repetitivos también han sido implicados en su producción ${ }^{18}$. Aproximadamente el $50 \%$ de las fracturas del pisiforme aparecen asociadas a otras lesiones de la extremidad superior, lo que dificulta su diagnóstico en la fase aguda. La proyección oblícua interna (muñeca en supinación de $45^{\circ}$ ), la proyección del túnel del carpo o la TAC son de ayuda cuando se sospecha una fractura del pisiforme. Es recomendable valorar la posible lesión del nervio ulnar debido a la proximidad en el canal de Guyon.
En la fase aguda, la inmovilización con yeso es recomendable pero no hay evidencia de que altere la historia natural de la fractura que puede evolucionar hacia una seudoartrosis o producir una artrosis piso-piramidal, que si son sintomáticas requerirán una escisión del pisiforme. Si el trazo de la fractura es transversal y existe una diástasis de los fragmentos, el tendón del flexor carpi ulnaris (FCU) estará roto; en estos casos la escisión del pisiforme a través de la rotura tendinosa, la reparación del tendón y la inmovilización en leve flexión e inclinación ciubital durante 3-4 semanas es el tratamiento recomendado.

\section{FRACTURAS DEL TRAPECIO}

Las fracturas aisladas del trapecio son infrecuentes y suelen estar asociadas a fracturas del primer metacarpiano y del radio distal ${ }^{16}$. Pueden ser consecuencia de un traumatismo directo o de un mecanismo indirecto por avulsión del ligamento transverso del carpo, por compresión axial del primer metacarpiano o por hiperextensión del pulgar.

La TAC, o en su defecto una proyección radiológica oblícua externa (muñeca en supinación de $45^{\circ}$ ) y/o del túnel del carpo, permiten diagnosticar estas fracturas que se clasifican en tres tipos. Fracturas del cuerpo; si no están desplazadas, la inmovilización incluyendo el pulgar durante 4 semanas es el tratamiento recomendado; si están desplazadas (el fragmento de mayor tamaño se desplaza, junto al metacarpiano hacia dorsal y proximal) es preciso realizar una reducción abierta y fijación interna con tornillos o Kirschner. Fracturas marginales trapecio-metacarpianas que se tratan mediante inmovilización incluyendo el pulgar durante 4 semanas. Fracturas de la tuberosidad que pueden localizarse en la base (Tipo I) y consolidan con inmovilización o en la punta (Tipo II) cuya evolución a la seudoartrosis es frecuente y si son sintomáticas requieren la escisión de fragmento. En los casos que evolucionan a una artrosis trapecio-metacarpiana o triescafoidea, la artrodesis o escisión-artroplastia son las opciones recomendadas. 


\section{FRACTURAS DEL TRAPEZOIDE}

Protegido por ligamentos potentes y rodeado por el trapecio, el hueso grande y el segundo metacarpiano, las fracturas aisladas del trapezoide son raras. Normalmente resultan de una fuerza indirecta aplicada en el eje del dedo índice y están asociadas a una fractura-luxación dorsal del segundo metacarpiano y/o del trapecio, o forman parte de una luxación axial del carpo ${ }^{16,19}$. Las fracturas-luxaciones palmares tienen una menor incidencia.

El diagnóstico se realiza mediante estudios radiológicos y TAC. Si no están desplazadas, la inmovilización durante 3-4 semanas es suficiente para conseguir la consolidación. Si existe desplazamiento es preferible realizar una reducción abierta y una fijación interna. En las lesiones crónicas, la artrodesis carpo-metacarpiana es la opción más recomendable.

\section{FRACTURAS DEL HUESO GRANDE}

Aparecen asociadas a lesiones óseas y/o ligamentosas de la muñeca en la mitad de los casos (fracturas-luxaciones perilunares o fracturas-luxaciones carpo-metacarpianas) $)^{16,20,21}$. Las fracturas marginales distales normalmente están asociadas a fracturas-luxaciones de la articulación carpo-metacarpiana de los dedos tercero y cuarto.

La TAC, la RM y los estudios radiológicos convencionales permiten realizar el diagnóstico de estas fracturas. En las fracturas sin desplazamiento, la inmovilización es suficiente para conseguir la consolidación. La reducción abierta y la fijación interna a través de un abordaje dorsal son la opción recomendada en las fracturas desplazadas. En los casos de seudoartrosis sintomáticas, las opciones de tratamiento comprenden la artrodesis mediocarpiana, el aporte de injerto óseo y fijación interna y la escisión-artroplastia con tendón.

\section{FRACTURAS DEL GANCHOSO}

Las fracturas del cuerpo pueden aparecer aisladas o asociadas a fracturas-luxaciones car- po-metacarpianas de los dedos cuarto y quinto o luxaciones axiales del carpo ${ }^{16-20} \mathrm{El}$ estudio radiológico puede alertar sobre la existencia de esta fractura y la TAC confirma el diagnóstico. Las fracturas aisladas y estables requieren un período de inmovilización de 4-6 semanas para su curación. Si la fractura está desplazada o está asociada a otras lesiones, precisa una reducción abierta y fijación interna.

Las fracturas de la apófisis unciforme son debidas a choques directos producidos por caídas o a impactos producidos por el mango de raquetas o palos de golf sobre la superficie cubital de la mano ${ }^{16,22}$. Los movimientos laterales y/o la flexión del quinto dedo contra resistencia incrementan el dolor a nivel de la apófisis unciforme. Se han descrito rupturas de los tendones flexores del quinto dedo por roce con los bordes de la fractura.

La proyección radiológica del túnel del carpo y la TAC permiten realizar el diagnóstico. En fase aguda, pueden consolidar con inmovilización pero debido a su precaria vascularización y a las fuerzas mecánicas de los tendones flexores en el túnel del carpo, que tienden a desplazar el fragmento hacia cubital cuando se flexiona el quinto dedo, estas fracturas tienden a evolucionar a seudoartrosis. La osteosíntesis puede considerarse en pacientes de alta demanda y en deportistas. En fase crónica, las alternativas terapéuticas contemplan la escisión del fragmento (Figura 7) o el aporte de injerto y la fijación interna $^{23}$.

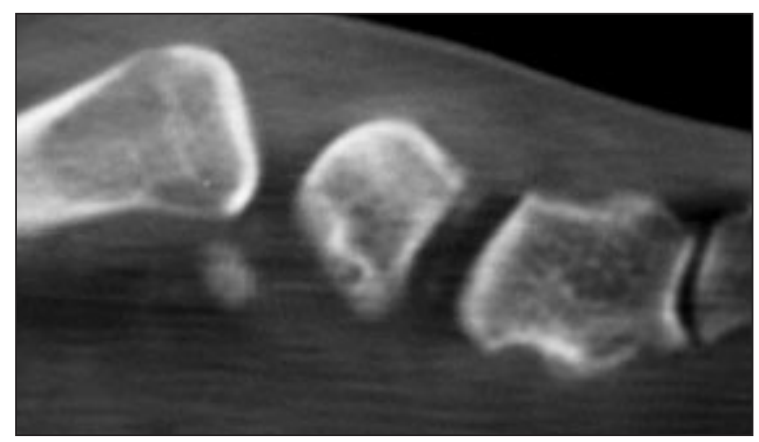

Figura 7: Seudoartrosis de la apófisis unciforme tratada con escisión del fragmento. 


\section{BIBLIOGRAFÍA}

1. Amadio PC, Moran SL. Fractures of the carpal bones. En: Green DP, Hotchkiss RN, Pederson WC, et al. Operative Hand Surg, 5th ed, vol 1. Philadelphia, PA: Elsevier Churchill Livingstone, 2005: 711-44.

2. Hove LM. Epidemiology of scaphoid fractures in Bergen, Norway. Scand J Plast Reconstr Surg Hand Surg, 1999; 33: 423-26.

3. Berdia S, Wolfe SW. Effects of scaphoid fractures on the biomechanics of the wrist. Hand Clin, 2001; 17: 533-40.

4. Gelberman RH, Menon J. The vascularity of the scaphoid bone. J Hand Surg Am, 1980; 5: 508-13.

5. Weber ER, Chao EY. An experimental approach to the mechanism of scaphoid waist fractures. J Hand Surg Am, 1978; 3: 142-8.

6. Herbert TJ, Fischer WE. Management of the fractures scaphoid using a new bone screw. J Bone Joint Surg Br, 1984; 66: 114-23.

7. Desai VV, Davis TR, Barton NJ. The prognostic value and reproducibility of the radiological features of the fractured scaphoid. J Hand Surg Br, 1999; 24: 586-90.

8. Schuind F, Haentjens P, Van Innis $F$ et al. Prognostic factors in the treatment of carpal sca- phoid nounions. J Hand Surg (Am) 1999; 24:761-76

9. Davis EN, Chung KC, Kotsis SV, et al. A cost/utility analysis of open reduction and internal fixation versus cast immobilization for acute nondisplaced mid-waist scaphoid fractures. Plast Reconstr Surg, 2006; 117: 1223-35.

10. Sanjuán R, Guillén M, García de Lucas F. Osteosíntesis percutánea de las fracturas de escafoides. Rev Iberamer Cir Mano, 2006; 2: 36-43.

11. Ashwin NR, Chung KC. Evidence-based management of acute nondisplaced scaphoid waist fractures. J Hand Surg Am, 2009; 34: 735-8.

12. Modi ChS, Nancoo T, Powers $D$, et al. Operative versus nonoperative treatment of acute undisplaced and minimally displaced scaphoid waist fractures-A systematic review. Injury, 2009; 40: 268-73.

13. Toby EB, Butler TE, McCormack TJ, et al. A comparison of fixation screws for the scaphoid during application of cyclical bending loads. J Bone Joint Surg Am, 1997; 79: 1190-7.

14. Teisen H, Hjarbaek J. Classification of fresh fractures of the lunate. J Hand Surg Br, 1988; 13: 458-62.

15. Höcker K, Menschik A. Chip fractures of the triquetrum.
Mechanism, classification and results. J Hand Surg Br, 1994; 195: 584-8.

16. Shah MA, Viegas SF. Fractures of the carpal bones excluding the scaphoid. J Am Soc Surg Hand, 2002; 2: 129-40.

17. Mayfield JK. Mechanism of carpal injuries. Clin Orthop, 1980; 149: 45-54.

18. Fleege MA, Jebson PJ, Renfrew DL, et al. Pisiform fractures. Skeletal Radiol, 1991; 20: 169-72.

19. García-Elías M, Dobyns JH, Cooney WP 3rd, Linscheid RL. Traumatic axial dislocations of the carpus. J Hand Surg Am, 1989; 14: 446-57.

20. García-Elías M, Bishop AT, Dobyns JH, et al. Transcarpal carpometacarpal dislocations, excluding the thumb. J Hand Surg Am, 1990; 15: 531-40.

21. Vance RM, Gelberman RH, Evans EF. Scaphocapitate fractures: Patterns of dislocation, mechanism of injury, and preliminary results of treatment. J Bone Joint Surg Am, 1980; 62: 271-6.

22. Walsh JJ 4th, Bishop AT. Diagnosis and management of hamate hook fractures. Hand Clin, 2000; 16: 397-403.

23. Watson HK, Rogers WD. Nonunion of the hook of the hamate: an argument for bone grafting the nonunion. J Hand Surg Am, 1989; 14: 486-90. 